\title{
FIRST RESULTS OF THE IOT BASED 300 KW 500 MHZ AMPLIFIER FOR THE DIAMOND LIGHT SOURCE
}

\author{
Morten Jensen, Matt Maddock, Simon Rains and Alun Watkins, Diamond Light Source Ltd, \\ Rutherford Appleton Laboratory, Chilton, Didcot, Oxfordshire, OX11 0QX, UK \\ Juergen Alex, Marc Mueller (Thales Suisse SA, Branch Broadcast \& Multimedia, Spinnereistrasse \\ 5, P.O. Box, CH-5300 Turgi, Switzerland)
}

\begin{abstract}
We present the first RF measurements of the IOT based $300 \mathrm{~kW} 500 \mathrm{MHz}$ amplifier for the Diamond Light Source. Four $80 \mathrm{~kW}$ IOTs are combined using a waveguide combiner to achieve the RF requirement of up to $300 \mathrm{~kW}$ for each of three superconducting cavities for the main storage ring. The IOTs are protected by a full power circulator and a $300 \mathrm{~kW}$ ferrite RF load. This is the first time IOTs will be used for a synchrotron light source. This paper gives an overview of the design of the Thales amplifiers and IOTs with commissioning results, including measurements of key components and overall RF performance, following factory tests and the installation of the first unit.
\end{abstract}

\section{BACKGROUND}

Diamond [1] is a $3 \mathrm{GeV}$ synchrotron light source currently being constructed on Harwell Chilton Science Campus, next to the Rutherford Appleton Laboratory. The building and machine is scheduled to be ready to welcome the first users in January 2007 following machine and beam line commissioning during 2006.

\section{SYSTEM OVERVIEW}

The RF system will initially put into operation two, but ultimately three, $500 \mathrm{MHz}$ superconducting cavities. Each cavity is fed from one high power amplifier [2] supplied by Thales Suisse SA, Branch Broadcast \& Multimedia. Each amplifier is designed to deliver up to $300 \mathrm{~kW}$ of continuous radio frequency (RF) power at $500 \mathrm{MHz}$. This is achieved by combining the outputs of four $80 \mathrm{~kW}$ Inductive Output Tubes (IOTs). A high voltage power supply provides $36 \mathrm{kV}$ to four IOTs and the RF of each pair of IOTs is coupled into a hybrid waveguide combiner. The combined RF from two such pairs is combined using a third waveguide combiner. To improve the efficiency when operating with fewer than 4 IOTs the final part of the waveguide combiner includes a phase shifter. A $300 \mathrm{~kW}$ circulator and a $300 \mathrm{~kW}$ ferrite load placed between the amplifier output and each cavity protect the amplifier in case of significant reflected power. Additionally, each combining branch is equipped with coaxial reject loads ensuring good isolation between individual IOTs. Allowing for combination losses through the combiner, mismatches and dissipated power in the circulator, up to $300 \mathrm{~kW}$ is available for distribution to each superconducting cavity. Each IOT is driven by a
$500 \mathrm{~W}$ solid state amplifier. The drive amplifiers each have independent gain and phase control to account for differences in IOT performances and transmission lengths. Each amplifier chain can be operated independently and contains a fast RF switch and a low level RF system controlling the cavity frequency as well as the amplitude and phase of the RF output to the cavity. To improve the match between the cavity and the amplifier, a high power three stub tuner is inserted in the waveguide run to the cavity. Additionally, a waveguide switch is included, which can be used to short the waveguide, isolating the cavity during any amplifier testing or commissioning. Figure 1 shows the layout of the key components of the amplifier.

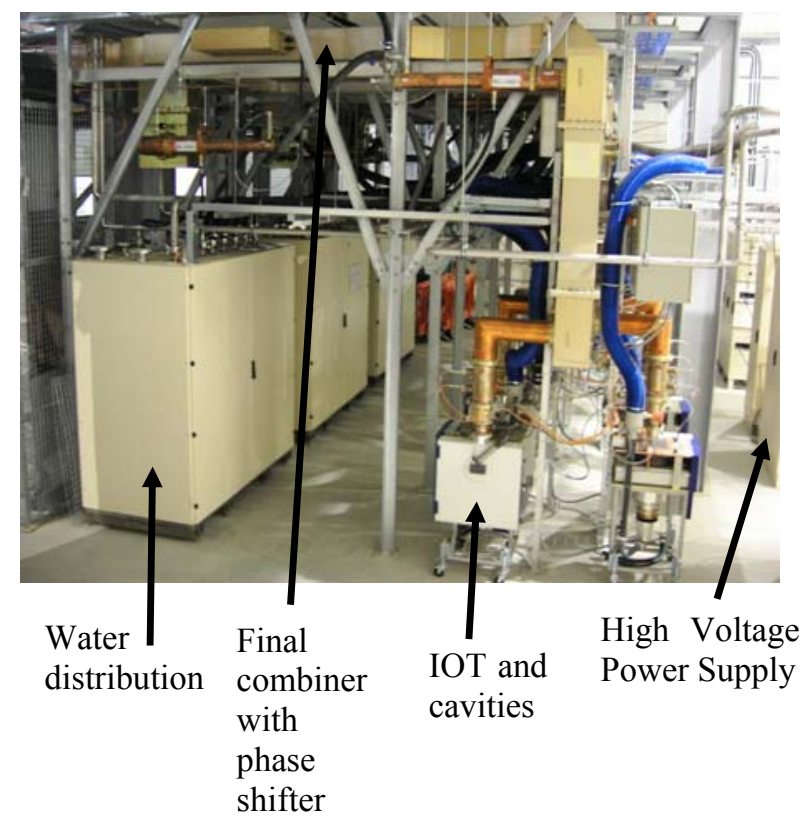

Figure 1: Photo of part of the RF installation showing IOTs with cavities, coaxial connection to waveguide combiner, the waveguide combiner running vertically up for the first combination and the horizontal section with phase shifter at the top of the picture. 


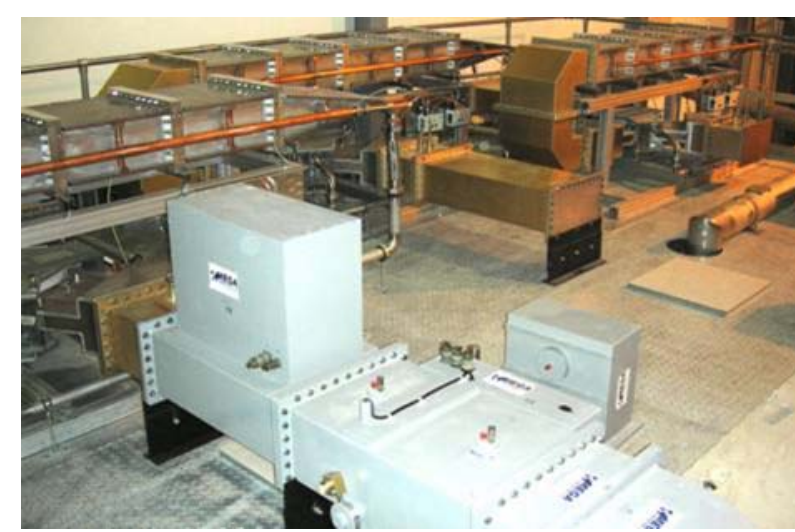

Figure 2: Photo of the high power circulator with $300 \mathrm{~kW}$ ferrite loads wrapped around the top. In the foreground the three stub tuner and the waveguide switch are visible.

\section{PROGRESS}

One amplifier was built and tested in the factory. During the testing some difficulties were encountered. These resulted in some delay and unfortunately also took the testing into a period where full power testing for extended periods was not possible. This is reflected in some of the results recorded. All three amplifiers have now been installed in Diamond. The low power commissioning, i.e. setting up of the control system, confirming signals and connections, has been completed and the high power set up and testing is scheduled to start in the middle of May 2005. The results presented here are from the factory testing.

\section{RESULTS}

Figure 3 shows the RF output power measured in the $300 \mathrm{~kW}$ ferrite load. This is following all combiner losses and following a double pass through the high power circulator. The RF power is calculated calorimetrically from the water flow and return to the water load. Both the inlet and outlet temperature $\left(\mathrm{T}_{\text {in }}\right.$ and $\left.\mathrm{T}_{\text {out }}\right)$ of the ferrite load were measured throughout the test, although the value of $\mathrm{T}_{\text {out }}$ was only recorded half way though the test. Approximately two hours into the test the power tripped due to some micro-arcing in the end of the ferrite load. The problem with micro-arcing resulted in a minor redesign of the ferrite tiles used. This result is the first evidence of an amplifier producing $300 \mathrm{~kW}$ of continuous $\mathrm{RF}$ power through the combination of four broadcast type IOTs.

In addition to the circulator protecting the amplifier in the event of significant reflected power, there are two further means of protecting the equipment and personnel. The first is a fast acting PIN diode in the RF chain and the second is the removal of the high voltage feed to the IOTs. To test the response time of the Thales amplifier to a short circuit event (eg an arc in the waveguide or in an IOT) a wire test was carried out. This was implemented by short circuiting the high voltage through a low resistance wire. The wire used in this case had a resistance of $0.295 \Omega$. Figure 4 below shows the response of the amplifier without the IOTs connected. The total energy dissipated in the simulated fault is $<0.3 \mathrm{~J}$. The amount of stored energy with the IOTs connected is typically still below $2 \mathrm{~J}$, which is comfortably within the specification and will not cause any damage to equipment.

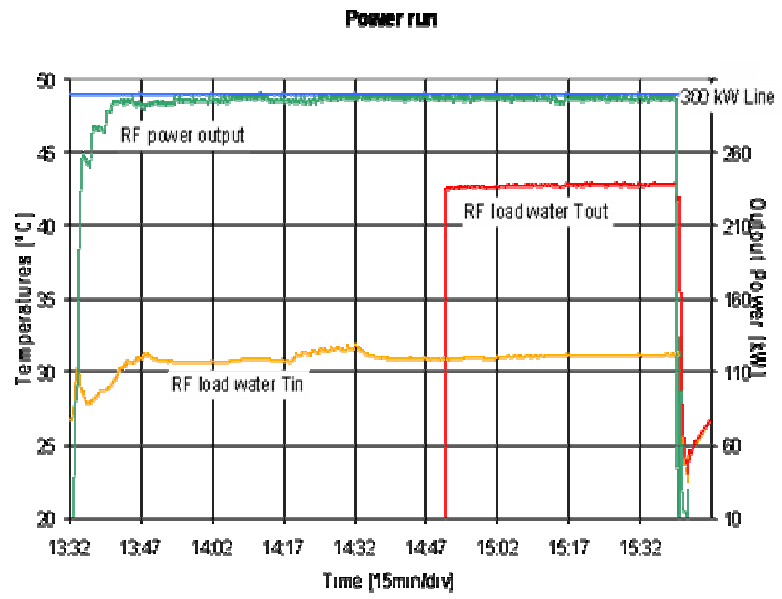

Figure 3: Plot of the output power over a two hour power run recorded on 23/11/04.

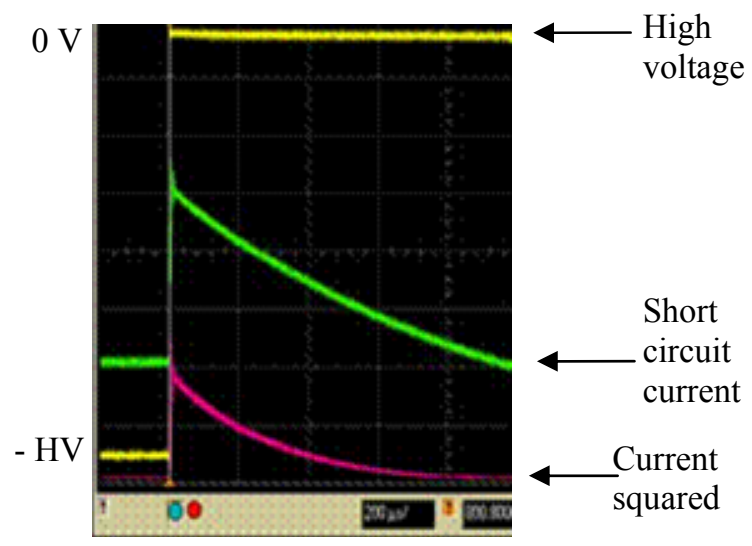

Figure 4: Plot of fault current during a simulated short circuit (Scale $5 \mathrm{kV} /$ division; $20 \mathrm{~A} /$ division; $200 \mu \mathrm{s} /$ division).

The measured power supply ripple is shown in Figure 5. This was measured under a load of 12.5 A (i.e. 4 IOTs producing $300 \mathrm{~kW}$ of RF output power). A high voltage divider with a 1000:1 ratio was used. The measured ripple was approximately $15 \mathrm{~V}_{\mathrm{rms}}$ and $100 \mathrm{~V}_{\text {peak-to-peak. }}$ We anticipate that the resulting cavity voltage ripple, as seen by the electron bunches, can be reduced further using an amplitude control loop implemented in the Low Level RF control system. 


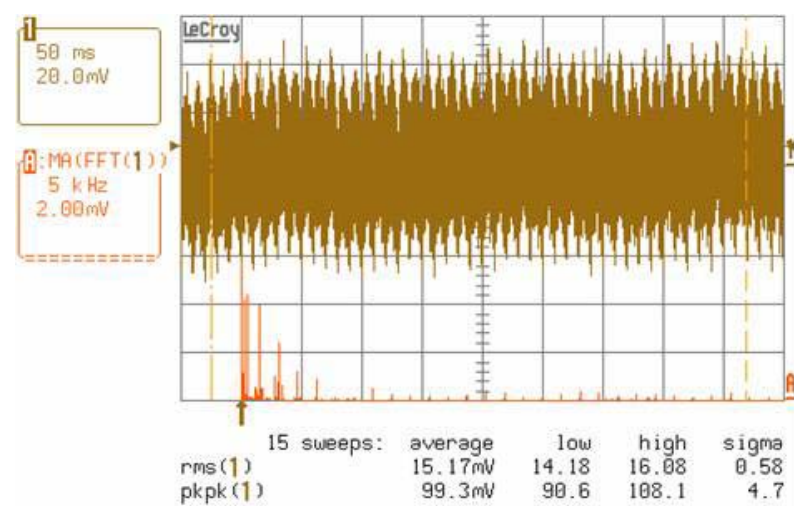

Figure 5: Output ripple of the amplifier under load.

Figure 6 shows the spectrum $\pm 0.5 \mathrm{MHz}$ from carrier. Each division corresponds to $100 \mathrm{kHz}$. The $50 \mathrm{kHz}$ and $100 \mathrm{kHz}$ lines are clearly visible on either side of the carrier albeit at least $70 \mathrm{~dB}$ below the $500 \mathrm{MHz}$ carrier.

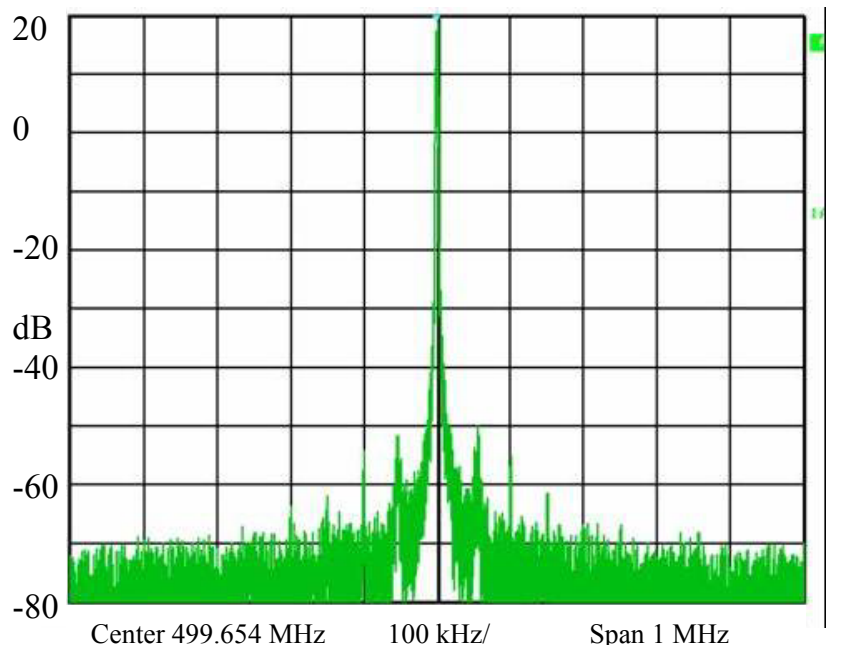

Figure 6: Spectrum of the output from the amplifier.

The frequency response of one of the IOT input cavities is shown in Figure 7 and the response of the IOT output cavity is shown in Figure 8.

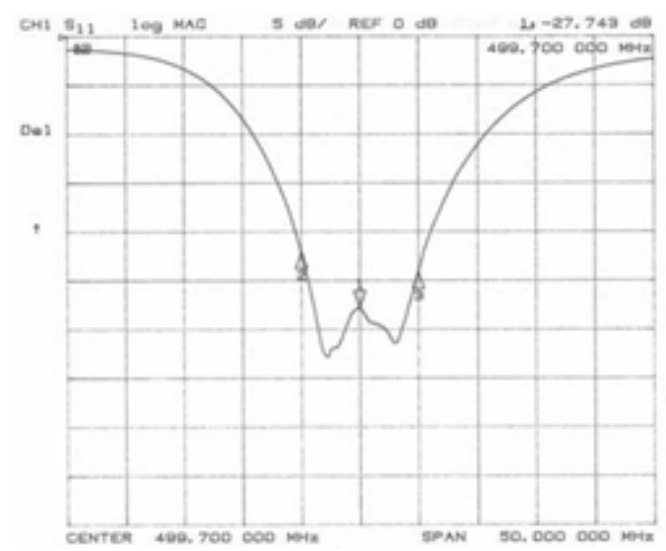

Figure 7. Typical S11 response of the input cavity (scale $5 \mathrm{MHz} /$ division; $5 \mathrm{~dB} /$ division).

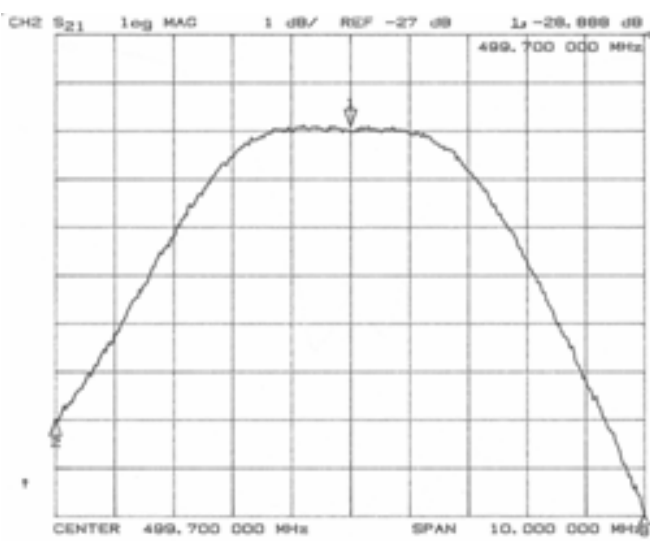

Figure 8: Typical S21 response of a tuned output cavity (scale $1 \mathrm{MHz} /$ division; $1 \mathrm{~dB} /$ division)

\section{DISCUSSION}

The preliminary factory results indicate positive evidence of the amplifier design. Four IOTs have successfully been combined to produce $300 \mathrm{~kW}$ of RF power at the output of the circulator after all combining losses, small mismatches and losses in the circulator. Whilst some micro-arcing in the ferrite load initially caused undesirable trips, the ferrite tiles have been redesigned and no further micro-arcing has subsequently been observed. The high power testing at Diamond is scheduled to start during May 2005. This testing will provide more evidence of gain, efficiency and overall amplifier performance.

\section{REFERENCES}

[1] R.P. Walker, "Progress with the Diamond Light Source Project", PAC 2003 Portland, Oregon, p 232.

[2] J. Alex et al, "Inductive Output Tube based $300 \mathrm{~kW}$ RF Amplifiers for the Diamond Light Source", EPAC 04, Luzerne, Switzerland, p 962. 